\title{
F-Shell Blob Model for a Tethered Polymer in Strong Flows
}

\author{
Diego Kienle ${ }^{t, \ddagger}$ and Walter Zimmermann*,‡ \\ FORUM Modellierung and Institut für F estkörperforschung, F orschungszentrum J ülich, \\ D-52425 J ülich, Germany; and Theoretische Physik, Universität des Saarlandes, \\ D-66041 Saarbrücken, Germany
}

Received September 28, 2000; Revised Manuscript Received August 31, 2001

\begin{abstract}
The F-shell blob model for tethered polymers as introduced recently by Rzehak et al. in E urophys. Lett. 1999, 46, 821 is investigated. In this model, each blob consists of a freedraining outer shell and a nondraining inner sphere in order to describe the partial draining of tethered polymers in uniform flow as found in computer simulations. It also covers the important property that hydrodynamic interaction effects depend on the shape of a polymer, and therefore, with increasing values of the flow velocities the model describes a transition from a partially draining, weakly elongated polymer, to a strongly stretched free-draining pol ymer at large flow velocities, similar as in simulations of bead-spring models. In the limit of a vanishing free-draining outer shell the F-shell blob model reduces to the model for nondraining blobs introduced by Brochard. It is shown that analytical solutions of blob models as obtained by an approximation of the vel ocity dependence of the end-to-end distance always have a larger slope than the exact solution which is obtained by numerical calculations. Moreover, a finite penetration of the flow into the polymer, as described by the F-shell blob model, reduces this slope further. This is in qualitative agreement with previous simulations of tethered bead-spring models in uniform flow, and therefore, large slopes as predicted previously by blob models cannot be expected in experiments.
\end{abstract}

\section{Introduction}

The behavior of dilute flexible polymers in flowing liquids is of practical and theoretical interest already for a long time and early models for single polymers in solution by Kuhn ${ }^{1}$ and Zimm² date back to the middle of the last century. Deformations of free and flexible polymers in solution are caused by the nonuniformity of the flow-field and vice versa, the dynamical deformation of a polymer induces a perturbation of the flow field. This interplay causes the non-Newtonian behavior of polymer solutions which is the origin of a number of spectacular flow effects. ${ }^{3}$ Only recently has experimental progress and numerical simulations contributed to a more detailed understanding of the interaction between polymer dynamics and the flow field, which has led to a renewed interest in the field. 4

In experiments using fluorescence microscopy, the deformation of polymers in flow has been visualized and analyzed. Examples are the stretching of tethered polymers in uniform flow which are held at one end by optical tweezers and the deformation of freely flowing polymers in shear and extensional flow. ${ }^{5-12} \mathrm{~A}$ powerful, theoretical tool to describe under external stresses the nonequilibrium state of polymers is the blob model, where the deformed chain is replaced by a sequence of independent and impenetrable blobs. Within this framework several scaling properties of polymers can be calculated for different external forces, such as polymers pulled at both ends, ${ }^{13-16}$ chains in shear flow ${ }^{17}$ or uniform flow, where one end is fixed, $16,18-20$ and polymer brushes. ${ }^{16,21,22}$

The appeal of blob models is, that analytical expressi ons can be derived, for instance for the shape and the el ongation of the tethered polymer in a flow field; e.g., the velocity dependence of the end-to-end distance of a nondraining polymer can be expressed by a simple

\footnotetext{
† F orschungszentrum J ülich.
}

‡ Universität des Saarlandes. power law $L(v) \propto v^{2} .{ }^{16}$ The analysis of the blob model leads also to a useful classification of the shapes of tethered polymers in four different velocity regimes. ${ }^{19}$ Starting at rather small vel ocities the polymer is still a spherical coil which is typical for the thermal equilibrium state. At intermediate velocities the so-called trumpet regime is reached, which is continued by the stem and flower regime at rather large velocities and ends up with the regime where the polymer has reached its fully stretched state.

Blob models depend crucially on several basic assumptions. In previous blob models one assumes first that all individual blobs are impenetrable and second that each part of a polymer inside a blob is in thermal equilibrium, so that the Flory relation $\mathrm{R}_{\mathrm{k}} \sim \mathrm{N}_{\mathrm{k}}{ }^{v}$ holds between the blob radius $R_{k}$ and the number of Kuhn segments $N_{k}$ of the respective part of the polymer bel onging to this blob.

The analytical derivation of the power laws for the polymer extension $L(v)$ and other quantities in the socalled trumpet regime involves a continuum approximation of the solution of the proper discrete recursion equation for the forces acting on the sequence of blobs. ${ }^{16}$ The trumpet regime applies only within a small velocity range, ${ }^{19}$ and the continuum approximation in this range gives a larger value for the power $\mu$ of the extension $\mathrm{L}(\mathrm{v}) \sim \mathrm{V}^{\mu}$ than obtained by a numerical solution of the full recursion equation of the blob model, as shown in this work in section III.

In experiments, the end-to-end distance of a tethered polymer in uniform flow is determined from temporally averaged data of fluorescently label ed DNA. ${ }^{5}$ During an averaging period the tethered DNA molecule performs orientational fluctuations as a whole as well as local conformational changes. Such fluctuations can also be investigated in simulations of a bead spring model for a tethered polymer. ${ }^{23}$ Moreover, and in contrast to experiments, in simulations al so the perturbation of the flow field can be determined besides the flow induced 
shape of the polymer. It turns out that the flow partially penetrates a tethered polymer, depending on the strength of the external flow. ${ }^{23}$ Because of this partial penetration, the assumption of impenetrable blobs in previous models is questionable.

To take the finite flow velocity at the mean location of the polymer into account, with the so-called F-shell bl ob model a generalization of the original nondraining blob model has been introduced. ${ }^{23}$ Within the F-shell model, the finite penetration of the flow into the polymer is described for each blob by a free-draining outer shell of constant thickness $d$ and a nondraining inner sphere.

This F-shell as well as previous blob models are defined in more detail in section II, where also some analytical results for blob models are presented. In section III, the numerical solution of the respective discrete recursion equations is compared with the analytical approximations as derived in section II. In addition, various quantities are determined, such as the polymer el ongation $\mathrm{L}(\mathrm{v})$ as function of the velocity $\mathrm{v}$ and the segment number $\mathrm{N}$, and the segment density is calculated as function of the distance from the tether point. Section IV contains the final remarks on the results.

\section{The F-Shell Blob-Model}

A polymer under an external stress may be described by a string of blobs, where the kth blob contains a subchain with a fraction $\mathrm{N}_{\mathrm{k}}$ of the $\mathrm{N}$ segments of the whole polymer. In addition, it is assumed that each blob by itself is in thermal equilibrium, so that the scaling relation ${ }^{15,24}$

$$
\mathrm{R}_{\mathrm{k}}=\mathrm{bN}_{\mathrm{k}}{ }^{v}
$$

between the diameter $R_{k}$ and the number of segments $\mathrm{N}_{\mathrm{k}}$ per blob may be applied. Here $\mathrm{b}$ denotes the length of a Kuhn segment and the exponent $v$ depends on the solvent quality. We only consider the two standard cases, i.e., $v=3 / 5$ for a good solvent, where the polymer resembles a self-avoiding random walk (SAW), and $v=$ $1 / 2$ for a $\Theta$-solvent. ${ }^{15,24-26}$

The radius $R_{k}$ and therefore the number of segments in a bl ob is determined by the balance between the total force $F_{k}$ acting on the kth blob and the thermal fluctuations, which is expressed by the Pincus rule $\mathrm{e}^{13,14}$

$$
\frac{\mathrm{k}_{\mathrm{B}} \mathrm{T}}{\mathrm{R}_{\mathrm{k}} \mathrm{F}_{\mathrm{k}}} \approx 1
$$

Tethered polymers in uniform flow are deformed due to the drag on monomers. The tension al ong the chain is nonuniform, and it increases from the free to the tethered end. Accordingly, at intermediate vel ocities the shape of a tethered polymer resembles a trumpet.6,16 In terms of the blob model, the force $F_{k}$ acting on the kth blob changes with $\mathrm{k}$ and increases from the free end to the tether point. For all three cases discussed here, i.e., for the free-draining, the nondraining, and the F-shell model the recursion relation for $F_{k}$ is different, as described in the following paragraphs.

Usually the hydrodynamic interaction between different parts of a polymer is taken into account in blob models by assuming impenetrable blobs, ${ }^{16}$ but the hydrodynamic interaction between blobs is discarded. However, recent simulations have shown that tethered polymers in uniform flow are partial draining, ${ }^{23}$ an effect which we model here with a generalized blob model, the F-shell blob model. To estimate the amount of partially draining we compare with a second limiting case, with the free-draining blob model, where independent of the conformation of the polymer, all of the segments experience the same drag force. ${ }^{23}$

A. The Force $\mathbf{F}_{\mathbf{k}}$ on a Nondraining Blob. The force exerted by the uniform flow with velocity $v$ on an impenetrable blob of radius $R_{k}$ is assumed to follow Stokes law $\mathrm{F}_{\mathrm{k}}=6 \pi \eta \mathrm{VR}$ for a sphere of the same radius $R_{k}$. (The prefactor $6 \pi$ is taken into account for completeness, but it is not crucial for the derivation of the scaling relations as discussed in this work.)

At the free end of the polymer, the first blob experiences only the Stokes drag, whereas the whole force acting on the second blob is the Stokes friction on it plus the tension induced by the friction on the first blob. Therefore, the total force acting on the kth blob counted from the free end is the sum of all Stokes forces acting on the blobs $\mathrm{j}=1, \ldots, \mathrm{k}$, i.e.

$$
\mathrm{F}_{\mathrm{k}}=6 \pi \eta v \sum_{\mathrm{j}=1}^{\mathrm{k}} \mathrm{R}_{\mathrm{j}}
$$

A combination of eqs $1-3$ then provides a recursion relation for the tension $\mathrm{F}_{\mathrm{k}}$

$$
\mathrm{F}_{\mathrm{k}}-\mathrm{F}_{\mathrm{k}-1}=6 \pi \eta \mathrm{v} \frac{\mathrm{k}_{\mathrm{B}} \mathrm{T}}{\mathrm{F}_{\mathrm{k}}}
$$

This may be solved by direct numerical iterations or analytically in the limit of large values of k, as described bel ow.

B. The Force $F_{k}$ on a Free-Draining Blob. For a free-draining blob model the friction force acting on the kth blob with $N_{k}$ beads is simply $N_{k}$ times the frictionforce, $6 \pi \eta \mathrm{av}$, acting on each single bead, where $\mathrm{a}$ is an effective bead radius specified later. Together this gives the total force acting on the kth blob

$$
\mathrm{F}_{\mathrm{k}}=6 \pi \eta \mathrm{va} \sum_{\mathrm{j}=1}^{\mathrm{k}} \mathrm{N}_{\mathrm{j}}
$$

Similar to the previous section II .A a recursion relation for the force $F_{k}$ acting on the kth free-draining blob is obtained by a combination of eqs 1,2 , and 5

$$
\mathrm{F}_{\mathrm{k}}-\mathrm{F}_{\mathrm{k}-1}=6 \pi \eta \mathrm{va}\left(\frac{\mathrm{k}_{\mathrm{B}} \mathrm{T}}{\mathrm{bF}_{\mathrm{k}}}\right)^{1 / v}
$$

C. The Force $\mathbf{F}_{\mathbf{k}}$ on a F-Shell Blob. Tethered polymers in uniform flow are neither free-draining nor completely impenetrable as has been shown by Rzehak et al. ${ }^{23}$ To describe such a partial-draining in the framework of blob models, the F-shell blob model for partial draining polymers has been introduced. I $n$ this model each blob is composed of a nondraining inner sphere and a free-draining outer shell of thickness d, as indicated in Figure 1.

For simplicity, we assume that the free-draining outer shell has a constant thickness $d$ in all blobs. Of course, this is an approximation, because the density of Kuhnsegments in a blob actually depends on the distance from the center ${ }^{26}$ Therefore, the penetration of the flow into a blob in principle also depends on the radius of this blob, but to the best of our knowledge no calcula- 


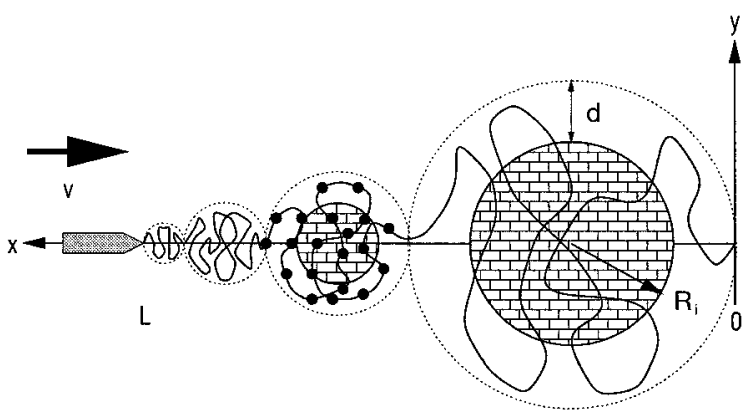

Figure 1. Sketch of a polymer tethered at one end and exposed to a uniform flow field with velocity $v$ in the $x$ direction. Within the blob model the deformed tethered polymer with $\mathrm{N}$ segments is approximated by a sequence of nonoverlapping spheres (blobs) of radius $R_{k}$ each containing $\mathrm{N}_{\mathrm{k}}$ Kuhn segments. In the F-shell blob model each blob consists of a free-draining outer shell of thickness $d$ and an impenetrable inner sphere (shaded).

tions about the penetration depth taking this fact into account are available yet. Nevertheless, the F-shell blob model with the assumption of a free-draining outer shell of constant thickness $d$ already qualitatively covers a crucial property of real polymers. For instance, at medium flow velocities rather long polymers are still coiled close to the free end and accordingly, they are nearly impenetrable in this range. Near the tethered end in contrast, the polymer may be already stretched and free-draining. Hence in the trumpet regime there is a transition from nondraining to the free-draining behavior along the string of blobs. This transition to partial draining may be tuned by the actual value of $d$.

For a blob with radius $R_{k}$ the radius of the nondraining inner sphere is

$$
\mathrm{R}_{\mathrm{k}}^{\text {non }}=\mathrm{R}_{\mathrm{k}}-\mathrm{d}
$$

so that due to the Flory relation 1 this inner sphere contains the number of Kuhn segments

$$
\mathrm{N}_{\mathrm{k}}^{\text {non }}=\left(\frac{\mathrm{R}_{\mathrm{k}}-\mathrm{d}}{\mathrm{b}}\right)^{1 / v}
$$

Accordingly, the number of segments in the freedraining shell is

$$
\mathrm{N}_{\mathrm{k}}^{\text {free }}=\mathrm{N}_{\mathrm{k}}-\mathrm{N}_{\mathrm{k}}^{\text {non }}
$$

Similar as for the two limiting cases discussed above, the total force $\mathrm{F}_{\mathrm{k}}$ acting on the kth blob in the F-shell bl ob model is the total force on the previous $k-1$ blobs plus the Stokes friction of the inner nondraining sphere, $6 \pi \eta \mathrm{VR}_{\mathrm{k}}^{\text {non }}$, together with the $\mathrm{N}_{\mathrm{k}}^{\text {free }}$ single bead frictions in the free-draining outer shell, i.e.

$$
\mathrm{F}_{\mathrm{k}}=\mathrm{F}_{\mathrm{k}-1}+6 \pi \eta \mathrm{VR}_{\mathrm{k}}^{\text {non }}+6 \pi \eta \mathrm{avN}_{\mathrm{k}}^{\text {free }}
$$

This together with the Pincus rule 2 and the scaling law in eql gives the recursion relation for the total force $F_{k}$ on the kth blob of the F-shell blob model:

$$
\begin{aligned}
\mathrm{F}_{\mathrm{k}}=\mathrm{F}_{\mathrm{k}-1}+6 \pi \eta \mathrm{v}\left(\frac{\mathrm{k}_{\mathrm{B}} \mathrm{T}}{\mathrm{F}_{\mathrm{k}}}-\mathrm{d}\right)+ \\
\frac{6 \pi \eta \mathrm{va}}{\mathrm{b}^{1 / v}}\left(\left(\frac{\mathrm{k}_{\mathrm{B}} \mathrm{T}}{\mathrm{F}_{\mathrm{k}}}\right)^{1 / v}-\left(\frac{\mathrm{k}_{\mathrm{B}} \mathrm{T}}{\mathrm{F}_{\mathrm{k}}}-\mathrm{d}\right)^{1 / v}\right)
\end{aligned}
$$

D. Boundary Condition for $\mathbf{F}_{\mathbf{k}}$. On the first blob at the free end with radius $R_{1}$ the total force $F_{1}$ is simply the local drag force acting on it. This requires the boundary condition

$$
\mathrm{F}_{0}=0
$$

for the recursion relations 4, 6, and 11 of the respective blob models.

E. Trumpet Regime. A string of blobs is formed only if the uniform flow is sufficiently strong and larger than some lower critical flow velocity $\mathrm{v}_{\mathrm{c} 1}$. Only if the Stokes force $F_{1}$ acting on the polymer becomes large enough, the radius due to the Pincus rule 2 becomes smaller than the value of the Flory radius of the unperturbed polymer $\mathrm{R}_{\mathrm{F}}=\mathrm{bN}^{v}$ :

$$
\mathrm{F}_{1}>\frac{\mathrm{k}_{\mathrm{B}} \mathrm{T}}{\mathrm{R}_{\mathrm{F}}}
$$

This inequality together with eqs 3 and 5 determines the lower critical velocity for the non- and free-draining limit as follows

$$
\mathrm{v}_{\mathrm{cl}}= \begin{cases}\frac{\mathrm{k}_{\mathrm{B}} T}{6 \pi \eta \mathrm{b}^{2}} \mathrm{~N}^{-2 v}: & \text { non-draining } \\ \frac{\mathrm{k}_{\mathrm{B}} T}{6 \pi \eta \mathrm{ab}} \mathrm{N}^{-(1+v):} & \text { free-draining }\end{cases}
$$

It is known from simulations of a polymer chain ${ }^{28}$ that a polymer will be more strongly elongated in the freedraining limit than for the case where hydrodynamic interactions are taken into account. Therefore, the critical velocity for the onset of the trumpet regime is smaller in the free-draining than in the nondraining limit, i.e., $v_{c 1}^{\text {free }}<v_{c 1}^{\text {non }}$. This observation provides a lower boundary for the effective bead radius a via

$$
\frac{\mathrm{v}_{\mathrm{c1}}^{\text {non }}}{\mathrm{v}_{\mathrm{c} 1}^{\text {free }}}=\frac{\mathrm{a}}{\mathrm{b}} \mathrm{N}^{1-v}>1
$$

The so-called trumpet regime is also restricted from above $\mathrm{v}<\mathrm{v}_{\mathrm{c} 2 .}{ }^{19}$ The upper critical velocity $\mathrm{v}_{\mathrm{c} 2}$ is reached when the tension along the chain close to the tether point, $F_{M}$, becomes so large, that the radius of the blob at the tether point becomes smaller than the Kuhn length $b: F_{M}>k_{B} T / b$. For velocities beyond $v_{c 2}$ there is a completely stretched part close to the tether point, the so-called stem, whereas at the other end the polymer coil has still the shape of a trumpet. This regime is the so-called stem and flower regime. For the free- and nondraining regime, one finds for the upper critical velocity:

$$
v_{c 2}= \begin{cases}\frac{k_{B} T}{6 \pi \eta b^{2}}\left(1+\sum_{j=1}^{k-1} N_{j}{ }^{\nu}\right)^{-1}: & \text { non-draining } \\ \frac{k_{B} T}{6 \pi \eta a b N}: & \text { free-draining }\end{cases}
$$

For the F-shell model the radii $R_{k}$ of the blobs must be larger than the penetration depth $d$, because the recursion relation eq 11 is only valid for $R_{k}>d$, whereby all previous blobs have still a nondraining core. Whenever $R_{k} \leq d$ one has to use beyond this value of $k$ the free- 
draining recursion eq 6 , which has to be solved for the kth and all the following free-draining blobs.

The velocity range in which the trumpet regime occurs, depends on the number $\mathrm{N}$ as can be easily seen for $v=3 / 5$ by expressing $\mathrm{v}_{\mathrm{c} 2}$ in terms of $\mathrm{v}_{\mathrm{c} 1}$

$$
\begin{aligned}
& v_{c 2}^{\text {non }}=N^{1 / 5} v_{c 1}^{\text {non }} \\
& v_{c 2}^{\text {free }}=N^{v} v_{c 1}^{\text {free }}
\end{aligned}
$$

Therefore, for a large number of segments $\mathrm{N}$ the vel ocity range of the trumpet regime is much larger in the freedraining limit than in the nondraining limit.

F. Numerical Procedure. A major issue of this work is the calculation of various quantities of a tethered polymer as a function of the flow vel ocity. The number of blobs increases with the flow velocity and it is assumed that in the trumpet regime each blob is filled. Therefore the total number of $\mathrm{N}$ Kuhn segments are distributed over $M$ blobs with $N_{k}$ segments in the kth blob and $k=1, \ldots, M$. With $M$ below some maximum number determined by the upper limit of the trumpet regime $\mathrm{v}_{\mathrm{c} 2}$, such a distribution of the $\mathrm{N}$ segments is only possible for one value of the flow velocity $v$ for each $M$. These discrete values of the flow velocity may be determined by using the conservation condition

$$
\mathrm{N}=\sum_{\mathrm{k}=1}^{\mathrm{M}} \mathrm{N}_{\mathrm{k}}
$$

whereby $N_{k}$ may be expressed in a more appropriate form as function of the velocity dependent forces, $F_{k}(v)$

$$
\mathrm{N}=\sum_{\mathrm{k}=1}^{\mathrm{M}}\left(\frac{\mathrm{k}_{\mathrm{B}} \mathrm{T}}{\mathrm{bF}_{\mathrm{k}}(\mathrm{v})}\right)^{1 / v}
$$

$F_{k}(v)$ itself is determined for all blob indices $k=1, \ldots$, $M$ and as a function of the flow velocity by using one of the recursion relations described above. For a given number of blobs $M$, the velocity is then iterated, for instance by a N ewton scheme, to fulfill the conservation condition in eq 18. If after termination of this iteration one of the blobs is smaller than the length b of a Kuhn segment, then the trumpet regime has been left and $M$ is already too large. With the velocity $\mathrm{v}(\mathrm{M})$ as function of the blob number $M$, the length $L$ of the polymer coil in the trumpet regime can be determined by the sum over all the blob radii $R_{k}$

$$
L(v)=\sum_{k=1}^{M} R_{k}=\sum_{k=1}^{M} \frac{k_{B} T}{F_{k}}
$$

Another interesting quantity is the local segment density $\rho_{\mathrm{k}}=\mathrm{N}_{\mathrm{k}} / \mathrm{R}_{\mathrm{k}}$, investigated later.

G. Analytical Solutions of the Recursion Relations. The recursion relations eqs 4 and 6 may be solved analytically in the two limits $k=1$ and $k \gg 1$ as shown in this section. Accordingly, a number of quantities which characterize the tethered polymer in a uniform flow may be calculated too. In this section II.G for the nondraining limit only polymers with $v=3 / 5$ are considered.

For the force on the first blob at the free end, $k=1$, one obtains with the boundary condition $\mathrm{F}_{0}=0$

$$
\mathrm{F}_{1}= \begin{cases}\left(6 \pi \eta \mathrm{vk}_{\mathrm{B}} \mathrm{T}\right)^{1 / 2}: & \mathrm{nd} \\ (6 \pi \eta \mathrm{av})^{v / 1+v}\left(\frac{\mathrm{k}_{\mathrm{B}} \mathrm{T}}{\mathrm{b}}\right)^{1 / 1+v}: \mathrm{fd}\end{cases}
$$

and for large values of $k$ one obtains with a power-law ansatz $\mathrm{F}_{\mathrm{k}} \propto \mathrm{Ak}^{\mathrm{w}}$ for the force at the kth blob

$$
\mathrm{F}_{\mathrm{k}}= \begin{cases}\left(12 \pi \eta \mathrm{vk}_{\mathrm{B}} \mathrm{T} \mathrm{k}\right)^{1 / 2}: & \mathrm{nd} \\ \left(6 \pi \eta \mathrm{av} \frac{1+v_{\mathrm{k}}}{v}\right)^{v / 1+v}\left(\frac{\mathrm{k}_{\mathrm{B}} \mathrm{T}}{\mathrm{b}}\right)^{1 / 1+v}: \mathrm{fd}\end{cases}
$$

It is often useful to express the force $F_{k}$ or the density of segments $\rho_{k}$ at the kth blob as function of the location $x$ with $x_{k}=\sum_{j=1}^{k} R_{j}$. Using the Pincus rule 2 together with the approximation 21 and replacing the sum by an integral one obtains

$$
\mathrm{X}_{\mathrm{k}}= \begin{cases}2\left(\frac{\mathrm{k}_{\mathrm{B}} \mathrm{T}}{12 \pi \eta \mathrm{v}}\right)^{1 / 2} \mathrm{k}^{1 / 2}: & \mathrm{nd} \\ (1+v)\left(\frac{\mathrm{bk}_{\mathrm{B}} T}{6 \pi \eta \mathrm{va} \frac{1+v}{v}}\right)^{v / 1+v} \mathrm{k}^{1 / 1+v}: \mathrm{fd}\end{cases}
$$

With the help of these expressions the index $\mathrm{k}$ can be eliminated, so that one obtains the force as function of the coordinate $x$

$$
F(x)= \begin{cases}6 \pi \eta v \mathrm{x}: & \mathrm{nd} \\ \left(\frac{6 \pi \eta v a}{\mathrm{vb}}\right)^{\nu} x^{\nu}: & \mathrm{fd}\end{cases}
$$

An expression for the segment density projected onto the $x$-axis, $\rho(x)$, follows from $\rho \approx N_{k} / R_{k}$

$$
\rho(\mathrm{x}) \propto \begin{cases}\left(\eta \mathrm{k}_{\mathrm{B}} \mathrm{T} \vee \mathrm{X}\right)^{\nu-1 / v}: & \mathrm{nd} \\ \left(\mathrm{k}_{\mathrm{B}} \mathrm{T}\right)^{\nu-1 / v}(\eta \mathrm{vX})^{\nu-1}: & \text { fd }\end{cases}
$$

Integrating the segment density $\rho(x)$ over the whole extension one finds from the conservation condition $\int_{0}^{L}$ $\mathrm{dx} \rho(\mathrm{x})=\mathrm{N}$ an expression for the extension $\mathrm{L}(\mathrm{v})$

$$
L(v) \propto \begin{cases}N^{3} v^{2}: & \text { nd } \\ N(N v)^{1-v / v}: & \text { fd }\end{cases}
$$

Evaluating the tension $\mathrm{F}(\mathrm{x})$ at $\mathrm{x}=\mathrm{L}$ provides the whole drag force acting on the tethered chain

$$
\mathrm{F}_{\mathrm{M}} \propto \begin{cases}\eta(\mathrm{N} \mathrm{v})^{3}: & \mathrm{nd} \\ \eta \mathrm{Nv}: & \mathrm{fd}\end{cases}
$$

\section{Results and Discussion}

Analytical expressions for the end-to-end distance and the density of Kuhn segments as function of the distance from the tether point have been derived previously for the nondraining blob model ${ }^{16}$ and for the free-draining one. ${ }^{28}$ In both cases a power-law ansatz has been used in order to solve the recursion relations for the nondraining, cf. eq 4, as well as for the free-draining blob model, cf. eq 6, respectively. In this section we compare these analytical results with the numerical solutions of eqs 4 and 6 . The numerical results for the F-shell blob model are described in section III.B. 

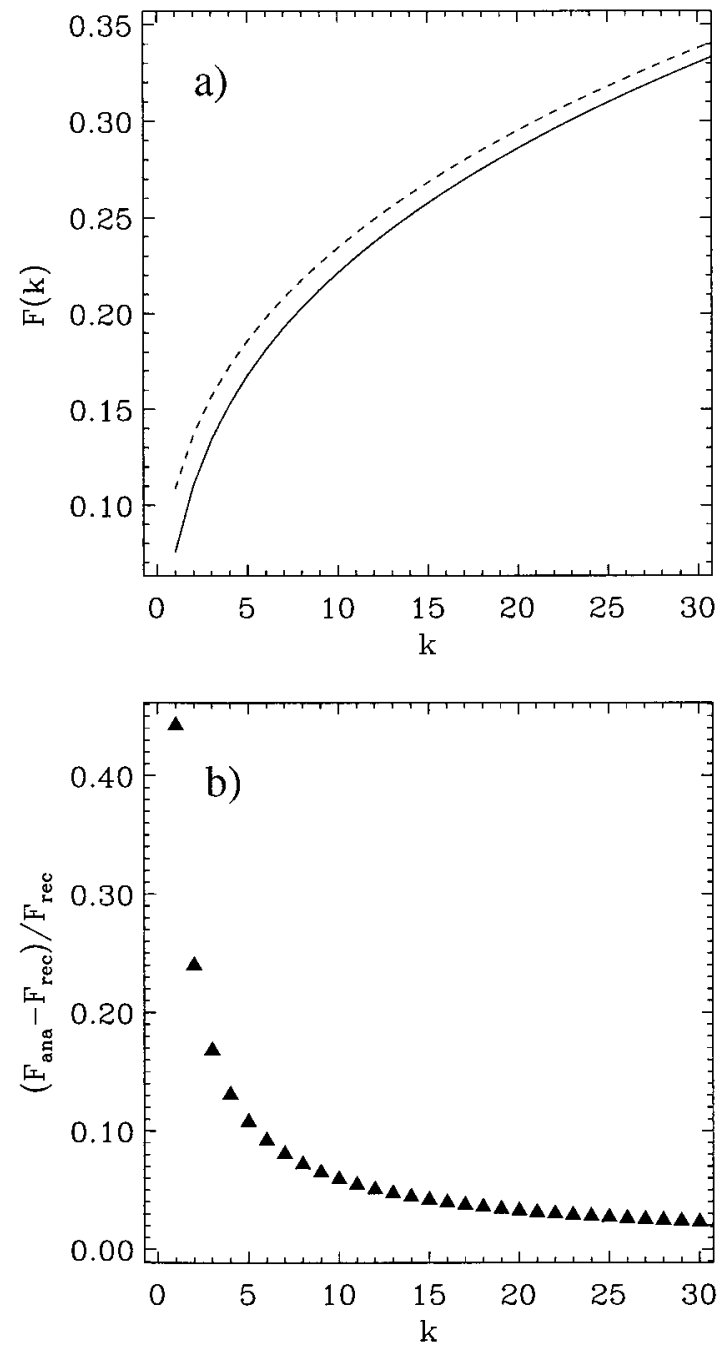

Figure 2. (a) Comparison for a free-draining blob model of the force $F_{k}$ acting on the kth blob, as given analytically in eq 21 (dashed line) with the numerical solution (solid line) of the recursion relation given in eq 6 , but only for the blobs with index number $k=1, \ldots, 30$ close to the free end. (b) Relative differences between the analytically and the numerically determined values for $F_{k}$ plotted. The flow velocity belongs to the trumpet regime with $\mathrm{v}_{\mathrm{c} 1}<\mathrm{v}=5.5 \times 10^{-4}<\mathrm{v}_{\mathrm{c} 2}$. The Flory exponent $v=1 / 2$ and the parameters $\eta=0.2, \mathrm{k}_{\mathrm{B}} \mathrm{T}=1.0, \mathrm{~b}=$ $1.0, a=0.22$ have been chosen.

A. Analytical vs Numerical Solutions of the Free- and Nondraining Blob Model. Only for long polymers and for intermediate values of the flow velocity, when the polymer shape is approximated by a huge number of blobs, large values of the blob index $k$ can be achieved. Only in this range of large values for $k$ is the power-law ansatz as given in eq 21 expected to become an exact solution of the recursion relation for the nondraining case given in eq 4 and the free-draining limit in eq 6.

To check this for the free-draining limit, we compare in Figure 2 the analytical solution of $F_{k}$, cf. eq 21 , with the numerical solution of the recursion relation in eq 6. Both, the analytical (dashed line) and the numerical solution (solid line) are plotted in Figure 2 for a chain with $\mathrm{N}=2 \times 10^{3}$ segments and a flow velocity of $v=5.5 \times 10^{-4}$, whereby $\mathrm{F}_{\mathrm{k}}$ is plotted only for the bl obs close to the free end with the blob index in the range $k=1, \ldots, 30$. Obviously, the analytical solution overestimates the exact solution of the recursion relation eq 6. The relative deviation between both decreases from

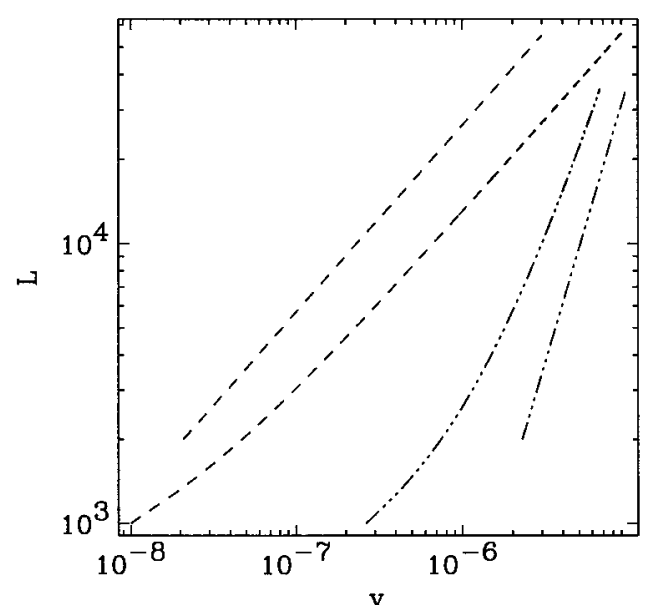

Figure 3. Elongation of a tethered polymer, $L(v)$, shown as a function of the velocity of a uniform flow for $\mathrm{N}=10^{5}$ segments of a free-draining (dashed) and a nondraining (dash-dotted) polymer model. In both cases the velocity has been restricted to the respective trumpet regime. The two straight lines correspond to the approximate analytical expressions given in eq 25. The other two curves are obtained by solving the recursion relation for $F_{k}$ numerically without further approximations and $\mathrm{L}(\mathrm{v})$ is obtained from $\mathrm{F}_{\mathrm{k}}$ via eq 19. $v=3 / 5$ is taken for the Flory radius and the bead radius $a=\xi / 6 \pi \eta=$ 0.265 has been chosen as in previous simulations. ${ }^{28}$

$\left(F_{1}^{\text {ana }}-F_{1}^{\text {rec }}\right) / F_{1}^{\text {rec }}=3^{1 / 3}-1$ to less than $2 \%$ at the blob number $k=30$. Hence for small values of $k$ close to the free end, the continuum approximation gives the largest error according to the discreteness of a chain of blobs.

If the forces $F_{k}=k_{B} T / R_{k}$ are calculated by the respective iteration schemes given above, the end-toend distance $L$ of the tethered polymer is obtained by the sum over the blob radii $R_{k}$, of. eq 19. The velocity dependence of the extension $L(v)$ is shown in Figure 3 for a rather long chain with $\mathrm{N}=10^{5}$ segments, whereby the dashed lines correspond to the free-draining limit and the dash-dotted lines to the nondraining limit. F or both cases $L(v)$ is plotted only in the respective trumpet regime $v_{\mathrm{c} 1}<\mathrm{v}<\mathrm{v}_{\mathrm{c} 2}$, whereby the analytical approximations for the critical velocities $\mathrm{v}_{\mathrm{c}}$ are given for $v=3 / 5$ by eq 14 and 16 . The straight lines in Figure 3 correspond to the analytical solutions as given by eq 25 . The difference between the slopes of the analytical and numerical solution becomes small only in a tiny velocity range near the upper limit of the trumpet regime, $\mathrm{v}_{\mathrm{c} 2}$, cf. Figure 3.

Especially at small flow velocities v, i.e., for a weak disturbance of the polymer, the numerically cal culated elongation $L(v)$ shows a convex behavior in a log-log plot as illustrated in Figure 3 and it is a nonlinear function of the velocity in general. Therefore, a power law such as $L(v) \sim v^{\mu}$ may be a local approximation only.

The assumption of long polymers and large numbers of Kuhn segments $\mathrm{N}$ is crucial in order that the derived power law $\mathrm{L}(\mathrm{v}) \sim \mathrm{V}^{\mu}$ becomes an acceptable approximation. ${ }^{16,28}$ Therefore, a fit of a simple power law to the numerically calculated curves $L(v)$ may become reasonable with increasing numbers of segments $\mathrm{N}$. Hence, for large values of $\mathrm{N}$ the slope of the curve $\mathrm{L}(\mathrm{v})$ resulting from numerical cal culations comes possibly closer to the power of that of the analytical curve. To check this, the extension $L(v)$ has been calculated numerically for different values of $\mathrm{N}$ and for each value of $\mathrm{N}$ we made a two parameter fit to the extension with $L(v) \sim A v^{u}$ either to the whol etrumpet regime or only to the upper 

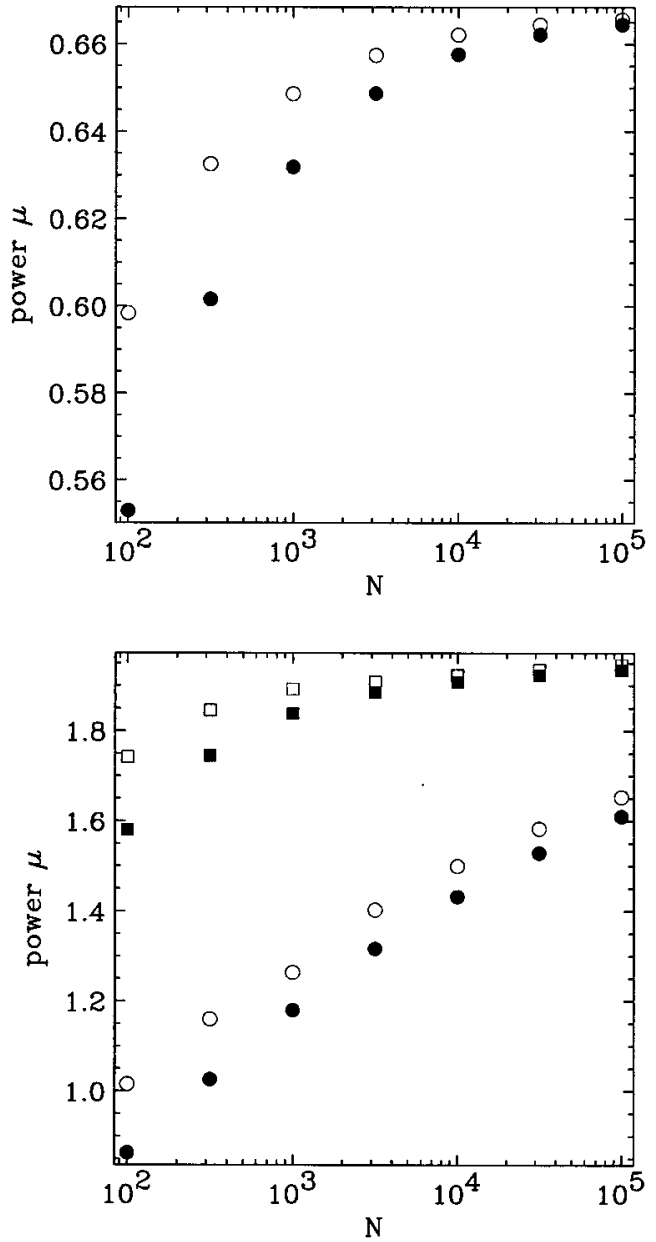

Figure 4. Numerically calculated extension L(v), as shown for instance in Figure 3 for $\mathrm{N}=10^{5}$, fitted by a two-parameter power law $L(v)=A v^{\mu}$ for several chains. The top part in this figure shows the $\mathrm{N}$-dependence of the fitted power $\mu$ for the free-draining blob model and the bottom part displays the power for the nondraining blob model. For both parts $v=3 / 5$ and the effective bead radius $a=\xi / 6 \pi \eta=0.265$. $\mu$ is either determined by a fit to the whole curve in the trumpet regime (filled circles) or to the upper quarter of the trumpet regime close to $v_{\mathrm{c} 2}$ (open circles). F or the nondraining case (squared symbols) the corresponding values for a three-parameter power-law $L(v)=A\left(v-v_{0}\right)^{\mu}$ has been included.

quarter of the trumpet regime, in the range $\mathrm{v}_{\mathrm{c} 1}+3\left(\mathrm{v}_{\mathrm{c} 2}\right.$ $\left.-v_{\mathrm{c} 1}\right) / 4<v<v_{\mathrm{c} 2}$. The values for the power $\mu$ obtained by these two types of fits are shown as function of the number of segments $\mathrm{N}$ in Figure 4.

The value of the analytically predicted power in the free-draining limit $\mu=2 / 3$ is rather quickly approached if the number of segments exceeds $\mathrm{N} \sim 10^{4}$ for both types of fits. On the other hand, the analytically predicted power $\mu=2$, cf. eq 25, for the nondraining limit is still not approached by such fits even for $\mathrm{N}=$ $10^{5}$ segments, cf. lower part in Figure 4.

F or velocities close to the upper vel ocity boundary of the trumpet regime, the number of blobs is large. Therefore, the difference between the analytically and the numerically determined force, $F_{k}^{\text {ana }}-F_{k}^{r e c}$, becomes small for large values of $k$, as can be seen in Figure 2. Accordingly, the difference between the analytically determined power and the power obtained from a fit with the power law $\sim \mathrm{V}^{\mu}$ to the numerically calculated extension $L(v)$ is expected to become small. I ndeed, for the free-draining limit the analytical result $\mu=2 / 3$ is

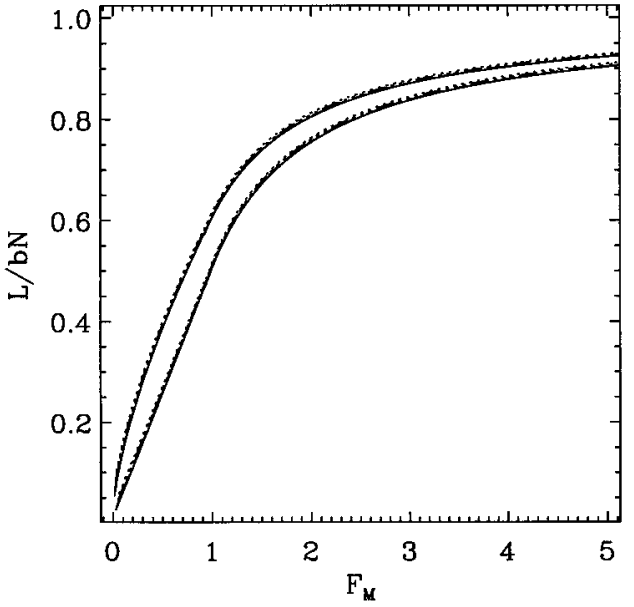

Figure 5. Fractional extension $\mathrm{L} / \mathrm{Nb}$ for a free-draining polymer shown as a function of total drag force $F_{M}$, whereby the two cases $v=1 / 2$ (lower curves) and $v=3 / 5$ (upper curves) for the Flory radius $\mathrm{R}_{\mathrm{k}}=\mathrm{bN}_{\mathrm{k}}{ }^{v}$ have been considered. F or each value of $v$ the fractional extension has been calculated for different numbers of Kuhn-segments, $\mathrm{N}=300$ (dotted), $\mathrm{N}=$ 500 (dash-dotted), $\mathrm{N}=1000$ (dashed), and $\mathrm{N}=1500$ (solid), but all of them fall on a simple master curve for each value of $v$. The effective bead radius has been chosen as $a=0.5$.

approached more quickly if $L(v)$ is fitted only in the upper quarter of the trumpet regime, cf. top part of Figure 3. On the other hand for the nondraining limit the analytically predicted power of $\mu=2$ is still not approached for both types of fits, even for the segment number $\mathrm{N}=10^{5}$. This may have several reasons.

One reason is that the trumpet regime is restricted to the velocity range $\mathrm{v}_{\mathrm{c} 1}<\mathrm{v}<\mathrm{v}_{\mathrm{c} 2}$, but for a reasonable good fit one would need $L(v)$ also in the range $0<v<$ $\mathrm{v}_{\mathrm{cl}}$. At $\mathrm{v}=0$, however, the end-to-end distance is nonvanishing with $L=R_{F}$. Therefore, it seems more appropriate to choose a three parameter fit which takes an offset into account such as $L(v)=A\left(v-v_{0}\right)^{\mu}$ or $L(v)$ $=A v^{\mu}+B$. In particular, for smaller values of $\mathrm{N}$, these formulas provide a value for the power $\mu$, which is closer to the analytically prediction as shown in the bottom part of Figure 4 for the nondraining limit (squared symbols). For larger values of $\mathrm{N}$ the offset as well as the weight of the nonlinear behavior of $L(v)$ at small values of $\mathrm{v}$ become less important for the fit and therefore the values obtained for $\mu$ become closer to those obtained from the two-parameter fits described above.

In the nondraining limit, there is another reason for the slower convergence of the power $\mu$ to the analytically determined value with increasing values of $\mathrm{N}$. The density of segments al ong a deformed tethered polymer, $\rho(x)$, is different for a free-draining and a nondraining model as shown in Figure 10. The distribution is steeper in the nondraining case and therefore the number of segments included in the blob at the free end is larger than in the free-draining case. As a consequence, the number of blobs for a given chain with $\mathrm{N}$ segments close to the upper limit of the respective trumpet regime, $\mathrm{v}_{\mathrm{c} 2}$, is larger for the free-draining case than for the nondraining case. E.g., for a chain with $\mathrm{N}=2000$ and $\mathrm{V}$ close to $v_{c 2}$, the number of blobs of a free-draining chain is nearly twice as much as for the nondraining chain. However, a larger number of blobs favors the convergence between the power of the analytical expression of $L(v)$ and the power fitted to the numerical curve. 


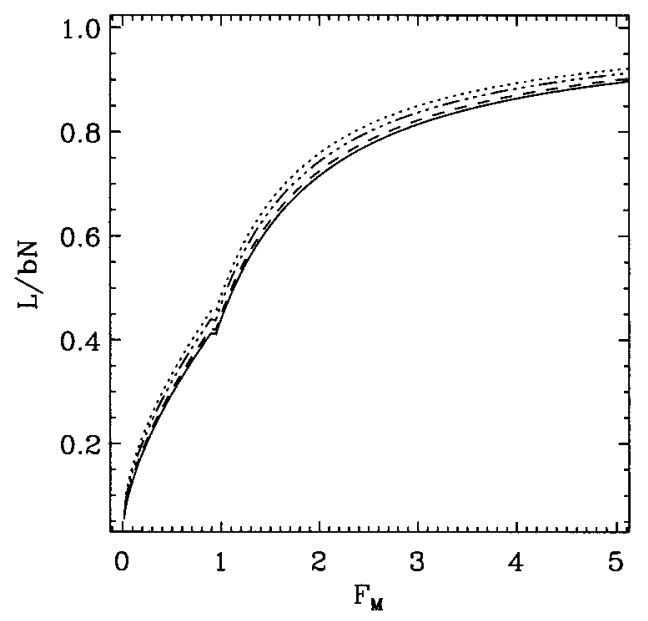

Figure 6. For a nondraining polymer with the Flory exponent $v=3 / 5$, the fractional extension $\mathrm{L} / \mathrm{Nb}$ shown as a function of the total drag force $\mathrm{F}_{\mathrm{M}}$ and for different chain lengths $\mathrm{N}=$ 300 (dotted), 500 (dash-dotted), 1000 (dashed), and 1500 (solid). The effective bead radius $a=0.5$ has been used along the free-draining stem.

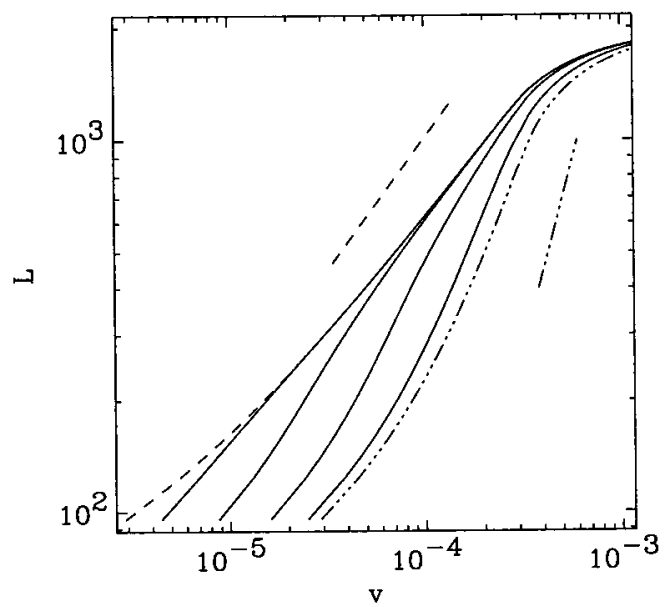

Figure 7. Elongation $L(v)$ of the polymer calculated for the $\mathrm{F}$-shell blob model with various values of the penetration depth $\mathrm{d}=1.0,5.0,15.0$, and 40.0 from right to left. The dasheddotted curve corresponds to the nondraining limit $(d=0)$ and the dashed one to the free-draining limit $\left(d=R_{F}\right)$. The straight lines are the power laws obtained from analytical approximations. The parameters are $\mathrm{N}=2000$ and $\mathrm{b}=1.0$ with $\mathrm{a}$ corresponding Flory radius of $\mathrm{R}_{\mathrm{F}}=\mathrm{bN}^{3 / 5}=95.6$. The bead radius has been chosen as $\mathrm{a}=0.48$.

The measurement or calculation of the extension $L$ of the tethered polymer as a function of the flow velocity is one way to characterize the interplay between the flow and the polymer deformation. For instance, since the extension $L(v)$ is different with and without hydrodynamic interaction the shape of this curve will give insight about the importance of the hydrodynamic interaction for a chain under consideration. The functional relationship $L(v)$ is also different with and without excluded volume effects $(v=1 / 2$ vs $v=3 / 5)$. In the free-draining limit and for $v=1 / 2$ the fractional extension $\mathrm{L} / \mathrm{Nb}$, which is obtained by the analytical expression in eq 25 , shows a universal linear velocity dependence $\mathrm{L} / \mathrm{Nb} \sim \mathrm{vNb}$ in the trumpet regime. For the case $v=3 / 5$ the universal nonlinear behavior $\mathrm{L}(\mathrm{v}) / \mathrm{Nb} \sim$ $(\mathrm{Nv})^{2 / 3}$ holds, whereas for the nondraining limit with $v$ $=3 / 5$ we find $\mathrm{L}(\mathrm{V}) / \mathrm{Nb} \sim(\mathrm{NV})^{2}$.

Another way to characterize the interaction between the flow and the polymer is to plot the extension vs the
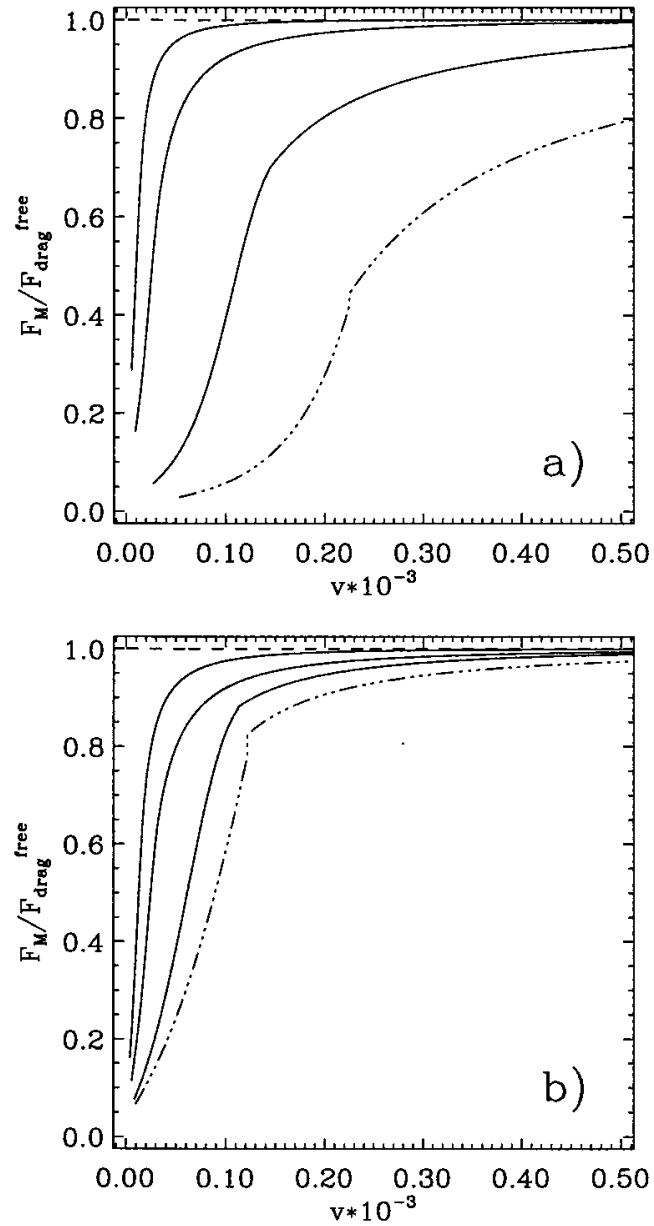

Figure 8. Total drag force $F_{M}$ normalized to the drag force in the free-draining limit, $F$ free, plotted as a function of the velocity $v$, in part a for $v=1 / 2$ and in part $b$ for $v=3 / 5$. The horizontal dashed curve is for the free-draining limit and the dash-dotted line is for the chain in the nondraining limit. The solid lines are for three different values for the penetration depth $\mathrm{d}$ of the $\mathrm{F}$-shell blob model. The parameters are $\mathrm{N}=$ $5000, a=0.5$, and for the solid lines $d=1.0,5.0$, and 10.0 from bottom to top.

drag force exerted by the flow on the tethered polymer. The drag force in the free-draining and nondraining cases is rather different, therefore this point of view may give rise to a different behavior. In the free-draining limit the drag force is just $\mathrm{N}$ times the single bead friction, $\mathrm{F}_{\mathrm{M}} \propto$ avN. Therefore, if $v=1 / 2$ the fractional extension varies linearly with the drag force, whereas for $v=3 / 5$ one has the behavior $\mathrm{L} / \mathrm{Nb} \sim \mathrm{F}_{\mathrm{M}}^{2 / 3}$. These two universal and analytical power laws are independent of the chain length and are valid within the trumpet regime. However, as can be seen in Figure 5 for several chains with different values of $\mathrm{N}$, for the numerical solution the master curve extends even beyond the trumpet regime into the stem and flower regime.

According to eqs 25 and 26 one expects in the trumpet regime for a nondraining polymer with $v=3 / 5$ a scaling for the fractional extension as $\mathrm{L} / \mathrm{bN} \propto \mathrm{F}_{\mathrm{M}}{ }^{2 / 3}$, independent of $\mathrm{N}$. This dependence on the drag force is the same as for the free-draining polymer with excluded volume effects $(v=3 / 5)$. Comparing this analytical result for the trumpet regime with the numerical solution of the recursion relations for different values of $\mathrm{N}$ and in the range $\mathrm{L} / \mathrm{Nb}<0.4$, as shown in Figure 6 , one does not find the same degree of agreement as for the free- 

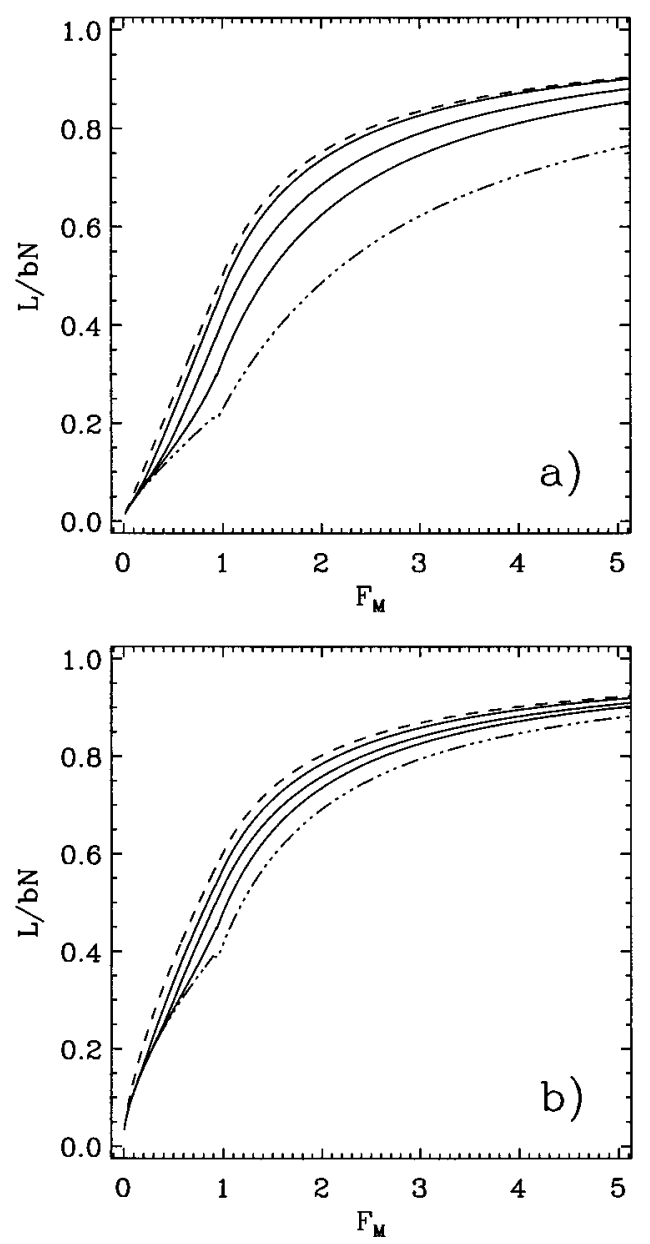

Figure 9. Fractional extension $\mathrm{L} / \mathrm{bN}$ as a function of the total drag force shown for the free-draining (dashed line) and the nondraining limit (dash-dotted line) and for the $\mathrm{F}$-shell blob model with $d=1.0,5.0$, and 10.0 (solid lines). The number of segments is $\mathrm{N}=5 \times 10^{3}$ and the effective bead radius $\mathrm{a}=$ 0.5 . In part $\mathrm{a}$, the Flory exponent is $v=1 / 2$, and in part $\mathrm{b}$, it is $v=3 / 5$.

draining limit. The differences become even larger beyond the trumpet regime in the stem and flow regime as also indicated in Figure 6.

In summary, at a given value of the vel ocity and also at a given value of the drag force, the extension $L(v)$ is larger in the free-draining limit than in the nondraining limit as shown in Figures 4-6.

B. F-Shell Blob Model. The vel ocity dependence of the extension $L(v)$ and other quantities change with the thickness $d$ of the free-draining outer shell of the F-shell bl ob model as described in this section. In Figure 7 the extension $L(v)$ is shown for a chain with $N=2000$ segments, $v=3 / 5$ and for four different values of $\mathrm{d}$ : $\mathrm{d}=$ 1.0, 5.0, 15.0, and 40.0 (solid lines). The curves $L(v)$ for a free-draining polymer (dashed line) and for the nondraining chain (dash-dotted), both of the same length as for the F-shell blob model, are included for comparison and the straight lines are the respective analytical curves as given by eq 25 . Note, for a vanishing velocity, the Flory radius for a self-avoi ding chain $(v=3 / 5)$ with $\mathrm{N}=2000$ Kuhn segments of length $\mathrm{b}=1$ is $R_{F}=$ $\mathrm{b}(2000)^{3 / 5}=95.6$.

With increasing values of $d$, the corresponding curves $L(v)$ deviate more and more from the nondraining case (dash-dotted line) and tend toward the free-draining limit (dashed line). In addition, L(v) approaches the

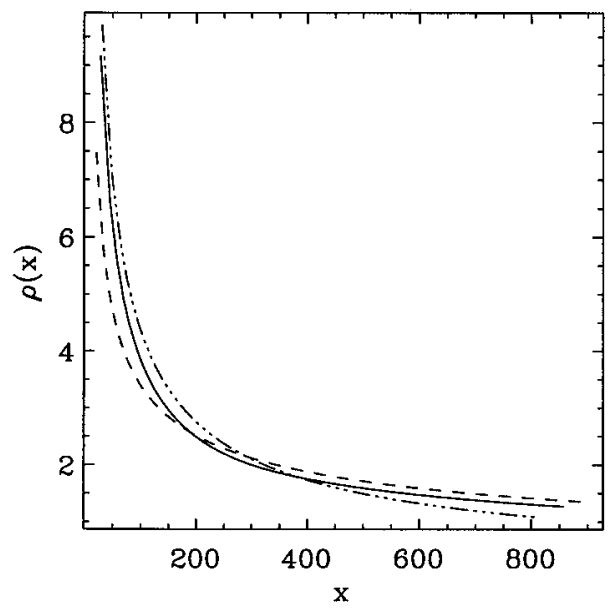

Figure 10. Segment density $\rho(x)$ shown as a function of the distance $x$ from the free end of the polymer for the free draining blob model (dashed), the nondraining blob model (dashed-dotted) and for the F-shell blob model for $\mathrm{d}=2.5$ (solid curve). The parameters have been chosen as in Figure 7: $\mathrm{N}=2000, \mathrm{~b}=1.0, v=3 / 5$, and $\mathrm{a}=0.48$, and the velocity has been adjusted such as to obtain in each case 360 blobs.

curve of the free-draining limit (dashed line) at first at large values of $v$ and with increasing values of $d$ also at smaller values of $\mathrm{v}$. I $\mathrm{n}$ the fully extended state at large values of $v$ the polymer is straight and free-draining. However, as long as v is not too large, there is already a number of small blobs close to the stem which become also free-draining due to the free-draining shell of thickness $d$. This means, that whenever one of the solid lines meets the curve of $L(v)$ for the free-draining limit, then even the largest blobs have become smaller than $\mathrm{d}$ and are finally free-draining.

The nondraining behavior of polymers is caused by the hydrodynamic interaction between different parts of a polymer. Due to the free-draining shell of the F-shell blob model the nondraining part is larger in the bigger blobs than in the smaller ones and therefore the relevance of the hydrodynamic interaction and the nondraining contribution changes along a tethered polymer. This variation of the nondraining contribution reduces the slope of $L(v)$ compared to the numerical solution of the blob model in the nondraining limit as shown in Figure 7. Note, in the latter case the curve obtained by a full numerical solution has already a smaller slope than the analytical formula given in eq 25, cf. Figure 3.

According to both of these effects one cannot expect in general the law $\mathrm{L}(\mathrm{v}) \propto \mathrm{V}^{\mu}$ with $\mu=2$ in experiments, as it has been predicted by the analytical solution of the nondraining blob model. ${ }^{16}$ Similar as the hydrodynamic interaction al so the excluded-volume effects in a tethered polymer are spatially dependent and are more relevant in the coiled part close to the free end than in the stretched part near the tether point. ${ }^{28}$ This spatial dependence of the excluded volume effect is not taken into account in the F-shell blob model, but it changes the slope of the curve $L(v)$ less strongly than the hydrodynamic interaction, as was shown in the numerical simulations by Rzehak et al. ${ }^{28}$

Figure 8 shows the total drag force $F_{M}$ exerted by the flow on the tethered polymer as a function of the velocity $v$ and for different values of $d$, whereby the drag force has been normalized to the drag force in the freedraining limit, $F_{\text {drag }}^{\text {free }} \sim$ aNv. These curves illustrate for 
both cases, $v=1 / 2$ and $v=3 / 5$, that the drag force acting on a nondraining polymer (dash-dotted lines) or on a partially draining polymer (solid lines) is al ways smaller than in the free-draining limit. Figure 8 also shows that the drag force in the nondraining limit converges quicker to that in the free-draining limit for a selfavoiding walk chain with $v=3 / 5$, cf. part b), than for $v$ $=1 / 2$, cf. part a). In part b) the drag force reaches nearly $80 \%$ of its value in the free-draining limit, already at the rather small velocity $v \sim 0.1 \times 10^{-3}$, whereas for $v$ $=1 / 2$ at the same vel ocity the relative drag force is only $5 \%$ of that in the free-draining limit. This happens due to a larger blob radius $R_{k}$ at the free end, which is caused by the excluded volume interaction. Larger values of $R_{k}$ enhance the viscous drag and induce therefore a stronger el ongation of the polymer than for an ideal chain $(v=1 / 2)$ in the nondraining limit. According to the smaller radii of the blobs of an ideal chain $\mathrm{R}_{\mathrm{k}} \propto \mathrm{N}_{\mathrm{k}}{ }^{v}$ with $v=1 / 2$, any finite penetration $\mathrm{d}$ changes the drag force with respect to the nondraining case in a much stronger manner than for a chain with $v=3 / 5$.

For the nondraining case the slope of the drag force as function of the velocity is discontinuous at the transition point from the trumpet to the stem and flower regime as can be seen in Figure 8 . In this case the discontinuity takes place for $v=1 / 2$ at $\mathrm{v}=0.22 \times 10^{-3}$ and for $v=3 / 5$ already at $v=0.12 \times 10^{-3}$. The discontinuity of the slope has the following origin. Within the blob model the stem of a polymer is freedraining and therefore it contributes to the drag force with a different slope $\propto v$ than the remaining nondraining part, which corresponds to the trumpet.

Therefore, beyond the trumpet regime in the range $v$ $>v_{\mathrm{C} 2}$ the velocity dependence of $F_{M}(v)$ changes its slope according to the increasing stem (free-draining part). F or the F-shell blob model with a free-draining shell of thickness $d$ there is already a free-draining part for every value of $v$ which contributes with a different slope to $F_{M}$ and therefore the transition to the stem and flower regime is smoothed (solid curves).

In Figure 9 we show the fractional extension $\mathrm{L} / \mathrm{bN}$ as function of the total drag force $F_{M}$ for a chain with $\mathrm{N}=$ $5 \times 10^{3}$ segments and different values of the thickness $\mathrm{d}$ of the free-draining shell.

Again the fractional extension at a given value of the drag force is larger for the free-draining than for the nondraining case. Also for this type of representation the changes with increasing values of $d$ are larger for the ideal chain with $v=1 / 2$ than for the self-avoiding random walk with $v=3 / 5$. The change in the slope of the dash-dotted line in Figure 9 (nondraining limit) marks again the onset of the stem and flower regime.

In Figure 10 the $x$-dependence of the segment density $\rho(x)$ is shown for the nondraining polymer model (dashdotted), a free-draining model (dashed) and for the $\mathrm{F}$-shell blob model with $\mathrm{d}=2.5$ (solid line). In all three cases a chain with $v=3 / 5$ and $\mathrm{N}=2000$ segments has been used and the coordinate $x$ measures the distance from the free end of the tethered polymer.

As already indicated by the previous figures, the extension $L(v)$ at a given velocity is larger in the freedraining case. To obtain for all three models a similar value for the extension the flow velocities have been chosen such, that the number of blobs for the three models is $M=360$. For the nondraining case this number corresponds to a rather stretched chain with the flow velocity close to the upper limit of the trumpet regime $v \sim v_{c 2}$. As indicated by the analytical formula in eq 24 the functional form of $\rho(x)$ is different in all three cases.

Since the number of segments $\mathrm{N}$ is fixed, which is formally given by $N=\int_{0}^{L} d x \rho(x)$, the two curves for the free- and the nondraining regi me must cross within the interval $0 \leq x_{c} \leq L$ which happens at $x_{c} \sim 300$ for the example shown in Figure 10. Note, when starting from the free end (left), the density in the nondraining limit (dash-dotted line) is always larger than in the freedraining case (dashed line). This behavior is related to the smaller drag force acting on a nondraining blob of the same size. The drag force on a nondraining blob increases linearly with $\mathrm{R}_{\mathrm{k}}$ and therefore like $\mathrm{N}^{v}$, whereas in the free-draining limit it increases linearly with $\mathrm{N}$ and therefore with $\mathrm{R}_{\mathrm{k}}{ }^{1 / v}$.

For finite values of $d$, the blobs become partially draining, so that the density at the free end is lowered with respect to the nondraining case, depending on the value of $\mathrm{d}$. On the other hand, at the tethered end, the curve is closer to the free-draining case (dashed). This behavior illustrates the crucial property of the F-shell blob model: The effects of the hydrodynamic interaction, manifested by the nondraining effect, change in space along the polymer. Therefore, it models a crucial observation in numerical simulations of tethered polymers, the partial draining effect.

\section{Conclusions}

Recently it became rather popular to describe the deformation of a tethered polymer in a uniform flow fiel $d$ by blob models. ${ }^{16,18-20,28}$ One reason for that is the possibility to derive with blob models analytical formulas for the major trends of the polymer deformation. The blob model is certainly a approximation for a real polymer which is reasonable only for rather long polymers. But to derive analytical formulas one needs even in the framework of the blob model further approximations. These approximations have been quantified in section III .A for the nondraining ${ }^{16}$ as well as for the freedraining blob blob model ${ }^{28}$ and a comparison with the full numerical solution of the blob model exhibits considerable deviations in some parameter range, as described in more detail in sections II.F. Figure 3, for instance, shows that the power 2 of the extension $L(v)$ $\propto \mathrm{v}^{2}$ as predicted for the nondraining polymer is too large when compared with the full solution of the blob model. According to this deviation al ready, the power $\mu$ $=2$ predicted by Brochard ${ }^{16}$ cannot be expected for chains with a rather small number of segments $\mathrm{N}<$ 3000 as used in experiments.

There are additional reasons for further deviations and which may further reduce this power $\mu$. In blob models for tethered polymers one assumes that the hydrodynamic interactions as well as the excluded volume interactions play the same role in every blob and therefore at every location al ong the deformed polymer in the trumpet regime. ${ }^{16}$ However, as shown recently by Rzehak et al., ${ }^{23}$ polymers are partially draining. This leads to a situation where small blobs close to the tethered end are al ready free-draining and near the free end nearly nondraining, depending on the thickness of the free-draining shell. Near the tether point, where only small blobs are expected, the excluded volume effect is also less important than in the larger blobs at the free end. Both effects will reduce the slope of the 
curve for the extension $L(v)$, as has been shown numerically. ${ }^{28} \mathrm{~F}$ or the applicability of the blob model one needs in addition very long polymers, but in experiments the available polymers include less than $\mathrm{N}=3000$ Kuhn segments, a number which is by far too small to show the predicted scaling even for an artificial model chain without excluded volume effects and without hydrodynamic interactions. ${ }^{27,28}$

Appropriate generalizations of the blob model may hel $p$ to avoid the described problems partially by keeping some of the mentioned advantages of blob models.

The F-shell blob model, for instance, as described in this work takes into account the variation of the effects of the hydrodynamic interaction along the deformed polymer in a rather simple but effective manner. Due to a free-draining outer shell of constant thickness $d$ a deformed tethered polymer described by this model changes from a partial draining behavi or near the free end of the polymer to a free-draining one at the tether point, and this happens al ready before the so-called stem and flower regime is reached. This spatially varying draining is in agreement with simulations of bead spring models and further results for this model, which are described in more detail in section III.B, tend in the same direction as found by numerical simulations. F or instance, the slope of $L(v)$ is reduced with increasing values of $d$.

Actually, the penetration depth of the flow into a coiled polymer is more complex as assumed by the F-shell blob model with the free draining shell of constant thickness $\mathrm{d}$. The penetration depth may be a function of the size of the blob, because the segment density is a function of the radius of a polymer coil. It may also depend on the flow velocity. Such possible refinements have been discarded here since they require a more sophisticated description of the coil of a polymer, e.g., in terms of a porous media. This will be considered in future work.

Here the stationary shape of a tethered polymer has been investigated for the F-shell blob model. The consequences of the free-draining outer shell for the relaxational dynamics, as it has been studied for the nondraining blob model ${ }^{18}$ or in simulations, ${ }^{29}$ are also interesting and will be discussed elsewhere.

Acknowledgment. It is a great pleasure to thank R. Rzehak and H. Müller-Krumbhaar for useful discussions.

\section{References and Notes}

(1) Kuhn, W. Kolloid-Z. 1934, 68, 2.

(2) Zimm, B. H. J . Chem. Phys. 1956, 24, 269.

(3) Bird, R.; Curtiss, C.; Armstrong, R.; Hassager, O. Dynamics of Polymeric Liquids I, II; Wiley \& Sons: New York, 1987.

(4) Nguyen, T. Q.; Kausch, H.-H. Flexible Polymer Chain Dynamics in Elongational Flow; Springer: Berlin, 1999.

(5) Perkins, T. T.; Quake, S. R.; Smith, D. E.; Chu, S. Science 1994, 264, 822.

(6) Perkins, T. T.; Smith, D. E.; Larson, R.; Chu, S. Science 1995 268, 83.

(7) Manneville, S.; Cluzel, Ph.; Voivy, J .-L.; Chatenay, D.; Caron, F. Europhys. Lett. 1996, 36, 413.

(8) Perkins, T. T.; Smith, D. E.; Chu, S. Science 1997, 276, 2016.

(9) Perkins, T. T.; Smith, D. E.; Chu, S. In Flexible Chain Dynamics; Nguyen, T. Q., Kausch, H.-H., Eds.; Springer: Berlin, 1997.

(10) Smith, D. E.; Chu, S. Science 1998, 281, 1335.

(11) Doyle, P. S.; Ladoux, B.; Voivy, J . L. Phys. Rev. Lett. 2000, 84, 4769.

(12) Meiners, J .-C.; Quake, S. R. Phys. Rev. Lett. 2000, 84, 5014.

(13) Pincus, P. Macromolecules 1976, 9, 587.

(14) Pincus, P. Macromolecules 1977, 10, 210.

(15) de Gennes, P. G. Scaling Concepts in Polymer Physics; Cornell University Press: Ithaca, NY, 1979.

(16) Brochard-Wyart, F. Europhys. Lett. 1993, 23, 105.

(17) Onuki, A. J. Phys. Soc. J pn. 1985, 54, 3656.

(18) Brochard-Wyart, F.; Hervet, H.; Pincus, P. Europhys. Lett. 1994, 26, 511.

(19) Brochard-Wyart, F. Europhys. Lett. 1995, 30, 387.

(20) Buguin, A.; Brochard-Wyart, F. Europhys. Lett. 1996, 29, 4937.

(21) Harden, J .; Cates, M. E. Phys. Rev. E 1996, 53, 3782.

(22) Milner, S. Science 1991, 251, 905.

(23) Rzehak, R.; Kienle, D.; Kawakatsu, T.; Zimmermann, W. Europhys. Lett. 1999, 46, 821.

(24) Doi, M.; Edwards, S. F. The Theory of Polymer Dynamics; Clarendon Press: Oxford, England, 1986.

(25) Flory, P. Statistical Mechanics of Chain Molecules; Interscience Publ.: New York, 1969.

(26) Yamakawa, H. Modern Theory of Polymer Solutions; Harper \& Row: New York, 1971.

(27) Rzehak, R.; Kromen, W.; Kawakatsu, T.; Zimmermann, W. Eur. Phys. J . E 2000, 2, 3.

(28) Rzehak, R.; Kienle, D.; Kawakatsu, T.; Zimmermann, W. In Molecular Dynamics on Parallel Computers, 1st ed.; Esser, R., Grassberger, P., Grotendorst, J., Lewerenz, M., Eds.; World Scientific: Singapore, 2000; p 120.

(29) Rzehak, R.; Zimmermann, W. Submitted to Phys. Rev. Lett. 2000.

MA0016883 